\title{
Direct Demodulation of Optical BPSK/QPSK Signal without Digital Signal Processing
}

\author{
TrongThuy HA, DongSun SEO \\ Dept. of Electronics, University of Myongji, 17058 Yongin, Korea \\ hatrongthuy93@gmail.com,sdsphoto@mju.ac.kr
}

Submitted June 13, 2018 / Accepted August 24, 2018

\begin{abstract}
We experimentally demonstrate the coherent detection of 5-Gbd/s BPSK/QPSK signal by direct phase compensation of the phase noise without using a sophisticated digital signal processing algorithm. The phase compensation is achieved by applying simply an error signal to a phase modulator located at the local oscillator for coherent detection, where the error signal is generated to keep the same power level for binary or quadrature signal.
\end{abstract}

\section{Keywords}

Phase noise, BPSK, QPSK, coherent detection, coherent optical communication

\section{Introduction}

In recent years, in order to fulfill the requirements of a high capacity communication, coherent optical modulation techniques based on multi-level amplitude and phase modulation have been extensively studied and applied as effective methods for future optical fiber communication systems [1-3]. The high-order phase and amplitude modulations enable high spectral efficiency (i.e., high bit rates) communication. However, the coherent detection still has some drawbacks. One of the most severe impairments that affect the coherent system employing high-order modulation formats is the phase noise [4], which is introduced by both transmitter and receiver lasers. The phase noises of the light sources, whose linewidths vary from several hundred $\mathrm{kHz}$ to $\mathrm{MHz}$, prevent correct detection of the transmitted data. The phase noise combined with amplitude noise also causes the imbalance of in-phase (I) and quadrature phase (Q), which degrade the performance of the coherent system. Consequently, many research groups investigated to remove and/or overcome the phase noise by adopting digital signal processing (DSP) techniques [5-8] and optical phase locked loops (OPLLs) [9-11]. Recently, the speed of DSP circuits is getting faster and faster to implement realtime compensation of the phase noise at the coherent receiver. However, coherent receivers based on DSP require complex and expensive electronics. On the other hand, to reduce the burden of the high-speed electrical digital signal processors at the demodulation of such coherent signals,
OPLLs or Costas loops using homodyne OPLLs have been applied widely [12-15]. Nevertheless, most coherent detections at higher symbol rates still require off-line DSPs.

In this paper, we propose a novel, simplistic method for the coherent detection of BPSK/QPSK signal. On contrast to conventional methods using complex DSP electronics [16], in the suggested method, the phase noise is suppressed directly by a phase modulator located at the local oscillator (LO) for coherent detection. This allows fast, real-time demodulation of coherently modulated signal, regardless of its symbol speed. To prove the proposed idea, coherent detection of binary and quadrature phase shift keying (BPSK and QPSK) signals have been demonstrated. We transmit BPSK and QPSK signals at 5 Giga bauds (or symbols) per second ( $\mathrm{Gbd} / \mathrm{s})$ through an optical channel and show eye diagrams and constellations for received signals, respectively. In addition, we also show corresponding simulation results based on our Optsim software.

\section{Experiments Setup and Results}

Figure 1 shows the schematic of our experiment setup for the generation and detection of QPSK optical signals. As a transmitter laser, a continuous wave (CW) external cavity laser (ECL) operating at $1550 \mathrm{~nm}$ with $100 \mathrm{kHz}$ of linewidth is used. The output of the ECL is divided into data and LO signals by a $3-\mathrm{dB}$ coupler. The data signal is quadrature phase-modulated by an optical I/Q modulator (Sumicem \# 32-16110) at $5 \mathrm{Gbd} / \mathrm{s}$. The optical I/Q modulator is driven by a pulse pattern generator (PPG) with a PRBS (pseudo-random binary sequences) pattern of length $2^{31}-1$. The in-phase (I) and quadrature-phase (Q) channels for the QPSK are implemented by applying data output and one-bit delayed data-bar output to an I/Q modulator, respectively. The BPSK modulation is achieved at the same setup by replacing the I/Q modulator by a simple phase modulator driven by the data output of the PPG. A polarization controller (PC) is used to feed 45 degree (to ensure the same power levels at the $\mathrm{X}$ and $\mathrm{Y}$ orthogonally polarized channels) polarized light to the coherent receiver. The data signals are then transmitted through a $10 \mathrm{~km}$ length of a G652D standard single mode fiber (SSMF). No in-line optical dispersion compensating fiber is used to compensate the chromatic dispersion. 


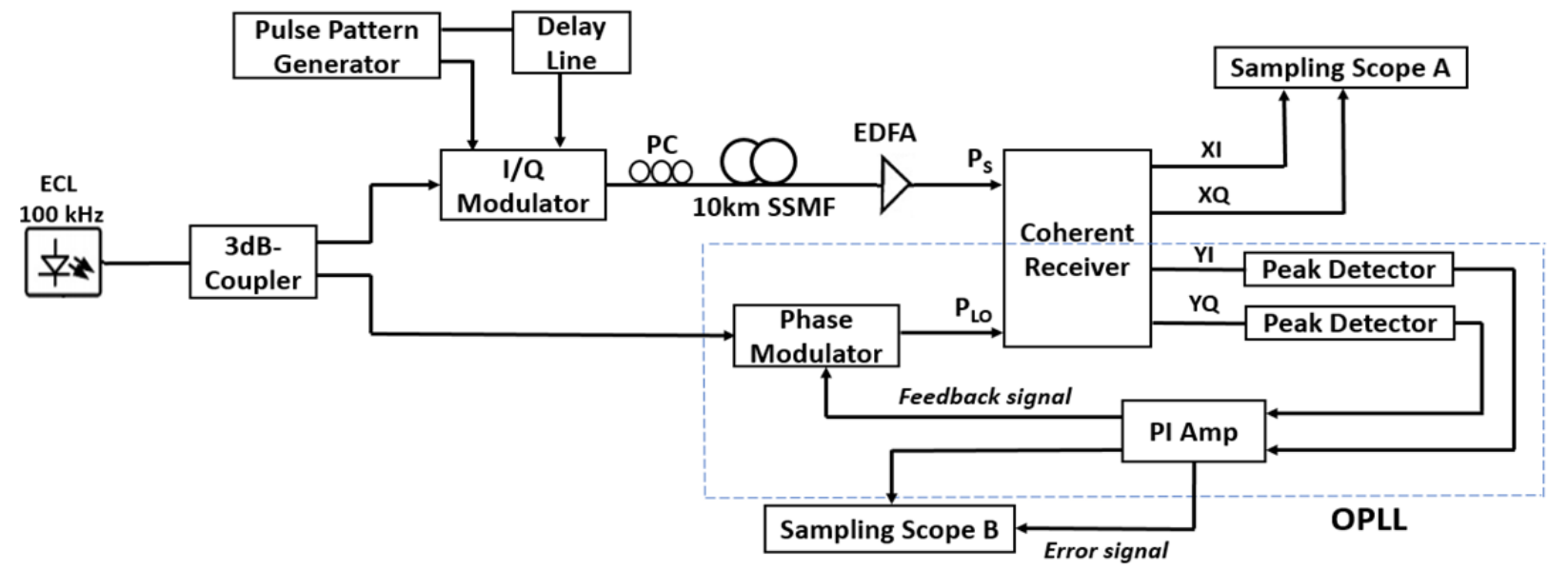

Fig. 1. Experiment setup.

An Erbium-doped fiber amplifier (EDFA) is used to keep the constant power level for both transmitted and back-to-back (B2B) signals at the receiver. Through the channel, the optical signal is attenuated by optical devices, such as $3 \mathrm{~dB}$-coupler ( $4 \mathrm{~dB}$ loss), I/Q modulator ( $7 \mathrm{~dB}$ loss), and SSMF with $0.2 \mathrm{~dB} / \mathrm{km}$ loss. The I and Q channels (XI and $\mathrm{XQ}$ ) of the $\mathrm{X}$-polarized signal are recorded by a fastdigital oscilloscope (Agilent 86100A). The other output (YI and YQ) for the Y-polarized signal are peak detected and fed to the positive and negative inputs of a proportional and integration amplifier (PI Amp, New Focus LB1005) to get the difference between the YI and YQ signal powers. The PI amp output (i.e., feedback signal) is applied to the phase modulator to adjust the phase of the LO signal. This leads to keep the power balance between YI and YQ channels for random data signals. The PI amp output shows the actual phase error signal to drive the phase modulator to compensate the phase noise. The phase error signal is monitored by the sampling scope B. The PI amp with optimum integration time provides a stable feedback control. Hence, the feedback loop works well in a simple and effective way to compensate any phase noise induced by the laser source and external turbulence (temperature, vibration, etc.) during the BPSK/QPSK signal transmission.

Figure 2(a) shows the eye diagram of 5-Gbd/s BPSK signal obtained from the direct PPG output. Figures 2(b) and (c) show the eye diagrams of the B2B detected signals without and with the phase noise compensation, respectively. Afterward, the persistent traces and data points are obtained for one minute. Mainly due to the phase noise of the laser source, the received eye pattern without applying our method is almost closed as shown in Fig. 2(b), indicating a high bit error rate (BER). By applying our phase noise compensation technique, the performance is improved significantly as proved by the clear eye opening shown in Fig. 2(c).

Figure 3 shows the induced phase error signals without (red line) and with (green line) feedback to compensate the phase noise. Without feedback, the magnitude of the phase error changes abruptly to saturate at the maximum level, indicating very large phase error (i.e., noise). With the feedback, the error signal shows negligible change, proving

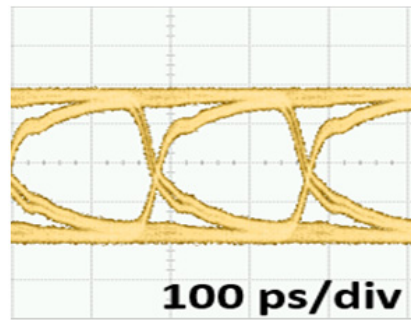

(a)

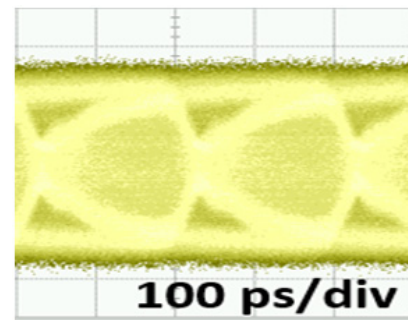

(b)

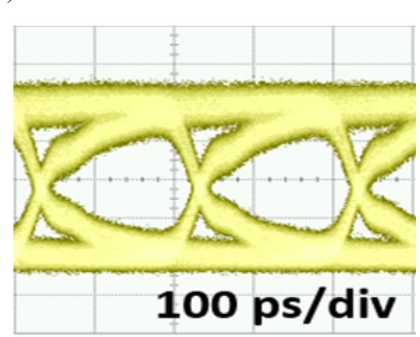

(c)
Fig. 2. Eye diagrams of $5-\mathrm{Gbd} / \mathrm{s}$ BPSK signal at $\mathrm{B} 2 \mathrm{~B}$; (a) PPG output, and detected signals without (b) and with (c) feedback, respectively.

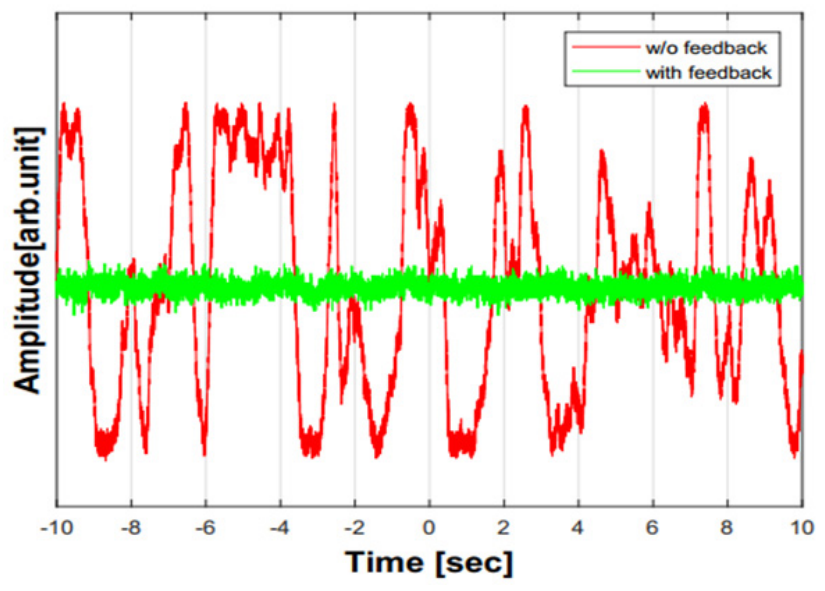

Fig. 3. Phase error signal to drive the phase modulator in Fig. 1. Red and green lines represent the signals without and with feedback, respectively.

that the feedback loop well tracks and compensates the phase noise. Moreover, the proposed scheme shows very fast locking time (determined by the feedback loop time 
constant) at the order of milliseconds, compared with a conventional method showing the order of 10 seconds [16]. Let's discuss a little more complicated case, the B2B detection of $5 \mathrm{Gbd} / \mathrm{s}$ QPSK signal. Figure 4 illustrates the eye diagrams of the $I$ and $Q$ channels without $(a, b)$ and with (c, d) our phase noise compensation, respectively. As expected, without phase noise compensation, the eyes are almost closed, indicating very poor BER performance. On the contrary, wide and clear eye openings can be seen by applying our phase noise compensation technique.

Figure 5 shows the corresponding constellations of Fig. 4, obtained by applying the I and Q signals into the horizontal and vertical inputs of the sampling scope $\mathrm{A}$ in Fig. 1. The constellation in Fig. 5(a) shows a typical random phase noise effect, rotating randomly the symbol points of the QPSK signal. After implementing the phase noise compensation, the symbol positions of the QPSK signal become stable as shown in Fig. 5(b). Note that the weak traces between the quadrature data points are observed due to the characteristics of the sampling scope measurement. Consequently, the phase noise compensation is confirmed clearly. In this way, the phase noise effect has been reduced without the aid of DSP algorithm.

Next we transmit the $5 \mathrm{Gbd} / \mathrm{s}$ QPSK data through the $10 \mathrm{~km}$ length of a G652D SSMF. Figures 6(a) and (b) show the measured eye diagrams of the I and Q channels of the received QPSK signals, respectively. Again our method works well to show reasonably good eye openings. Comparing to Fig. 4, slightly larger inter symbol interference (ISI) is observed. We think this increment is induced by the amplified spontaneous emission (ASE) noise of the EDFA (used to compensate the SSMF loss), enhancing the random

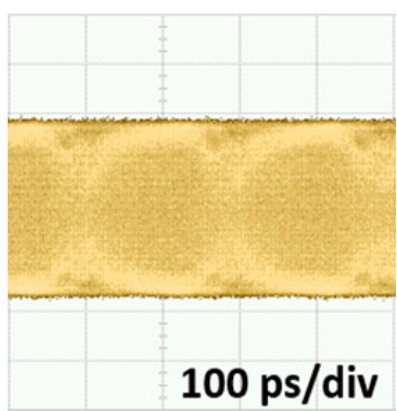

(a)

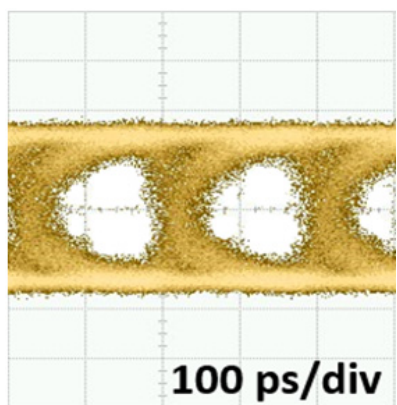

(c)

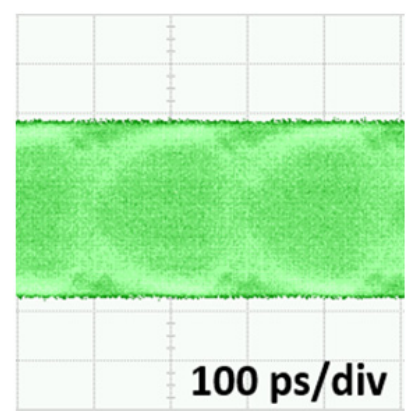

(b)

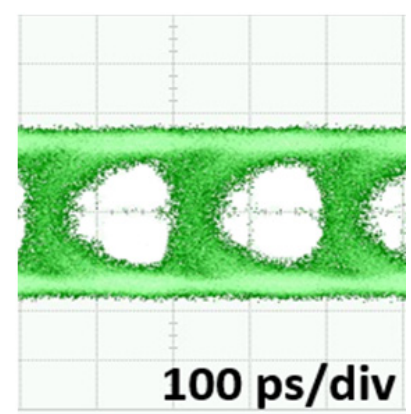

(d)
Fig. 4. Eye diagrams of $I$ and $Q$ channels of $5 \mathrm{Gbd} / \mathrm{s}$ QPSK signal at B2B; (a, b) without and (c, d) with phase compensation, respectively.

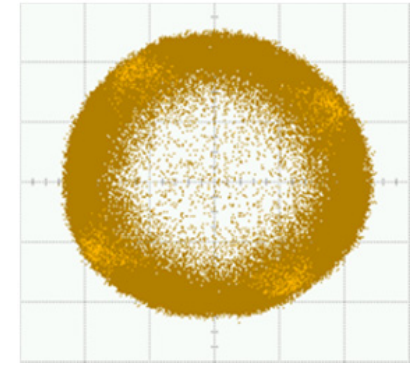

(a)

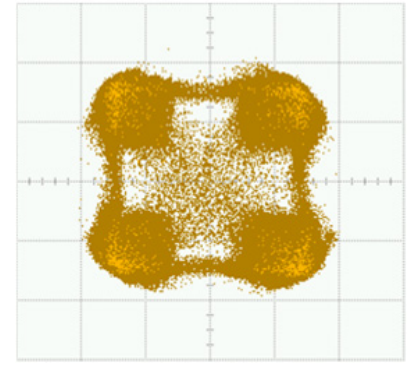

(b)
Fig. 5. Constellation diagrams for QPSK signal at B2B; (a) without feedback and (b) with feedback.

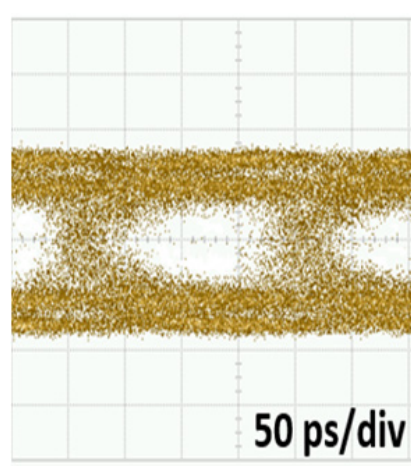

(a)

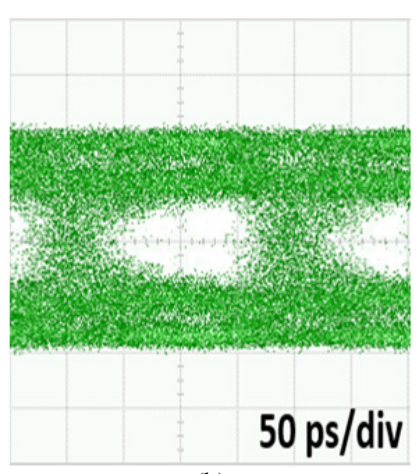

(b)
Fig. 6. Measured eye diagrams of the I (a) and Q (b) channel of $5 \mathrm{Gbd} / \mathrm{s}$ QPSK signal after $10 \mathrm{~km}$ transmission through SMF (with feedback method).

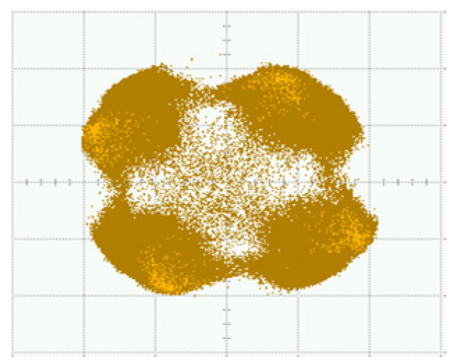

Fig. 7. Measured constellation of 5-Gbd/s QPSK signal obtained after $10 \mathrm{~km}$ SSMF transmission.

phase noise in the data signal. The accumulated random phase noise reaches sometimes beyond of the trackable range of our phase noise compensation loop. Additional signal distortion may also be induced by the fiber dispersion.

Figure 7 shows the corresponding constellations of the demodulated $5 \mathrm{Gbd} / \mathrm{s}$ QPSK signal after $10 \mathrm{~km}$ SMF transmission. As expected, the QPSK symbols can be distinguished from each other, demonstrating again the potential of our phase noise compensation method.

\section{Simulation and Results}

In order to prove the feasibility of the suggested method for longer distance transmission, we perform simulation based on our Optsim software package. Figure 8 presents the proposed implementation in the Optsim platform 


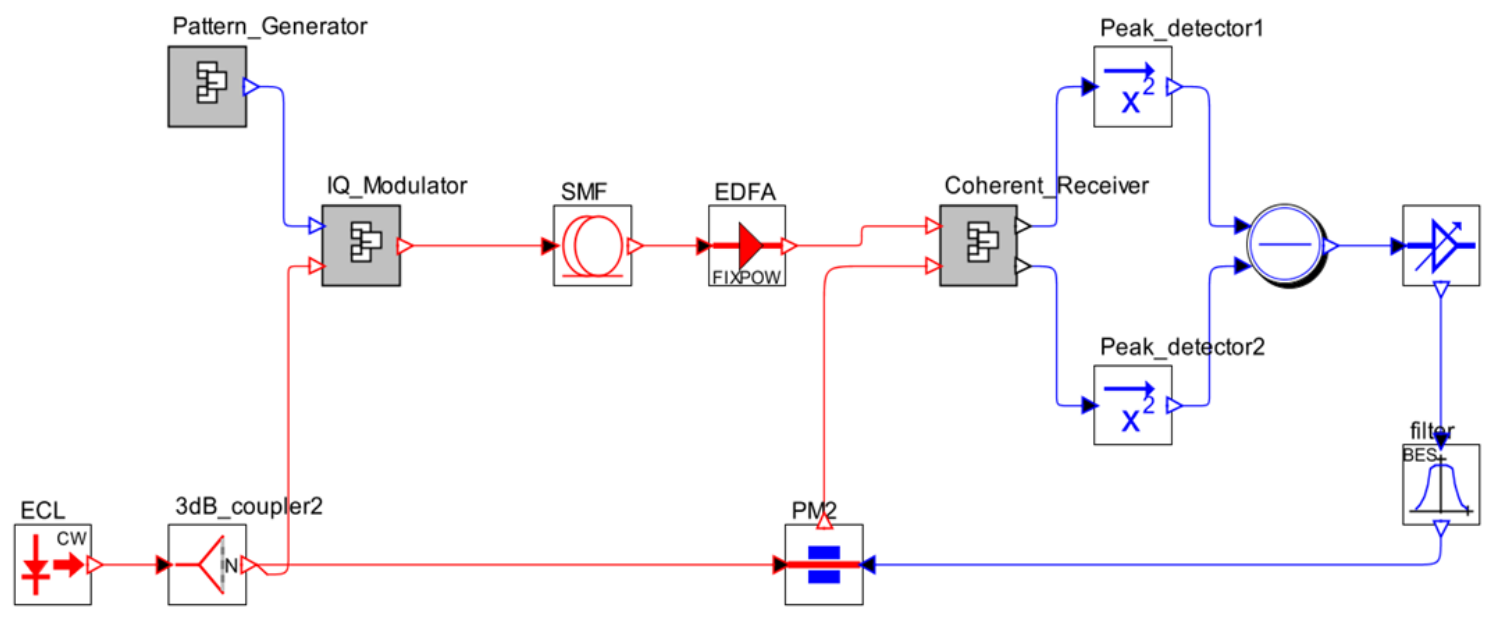

Fig. 8. Block diagram of the system used in simulation.

which is the same as the experimental setup shown in Fig. 1.

As we discussed, the laser source with $100 \mathrm{kHz}$ linewidth operates at $1550 \mathrm{~nm}$. The PRBS with length of the $2^{31}-1$ is applied to the I/Q modulator with two of MachZehnder (MZ) intensity modulators. The outputs from the $\mathrm{MZ}$ modulators have $\pi / 2$ phase difference to form the I and $\mathrm{Q}$ channels. The modulated signals are then transmitted over a standard G652 single mode fiber (SMF) with $0.2 \mathrm{~dB} / \mathrm{km}$ loss and $16 \mathrm{ps} / \mathrm{nm} / \mathrm{km}$ dispersion. The gain and noise figure of the EDFA are assumed as $3 \mathrm{~dB}$ and $5 \mathrm{~dB}$, respectively. The EDFA boosts the attenuated signal and matches the receiver input power to the experiments discussed above. The coherent receiver is consisted of four pin photodiodes with a quantum efficiency of $70 \%$ and responsivity is $1 \mathrm{~A} / \mathrm{W}$ followed by four transimpedance amplifiers. Total number of symbols is 32768 and each symbol is sampled by 26 times. The error signal is fed to the phase modulator at the rate of $\pi$ phase shift per $5 \mathrm{~V}$ to compensate the phase noise. Finally, in order to examine the output of the I and Q channels, scattering diagram and oscilloscope visualizer are installed as measurement systems.

The validity of our simulation results is confirmed by comparing them with the experiment results discussed above. Then, through the simulation, we show how the suggested idea would work for longer distance transmission.

Firstly, we investigate the eye diagrams and constellations of the transmitted QPSK signal at $5 \mathrm{Gbd} / \mathrm{s}$ at B2B detection, as shown in Figs. 9 and 10. As expected from the experimental results, the eye patterns are significantly affected by the laser phase noise, as shown in Fig. 9(a). On the contrary, the clear eye openings for both I and Q channels are observed, indicating effective phase noise reduction by the suggested method, as shown in Fig. 9(b).

Figure 10 shows the corresponding constellations of QPSK signal without (a) and with (b) phase compensation. The phase noise blurs and rotates randomly the symbol points of the QPSK signal, as shown in Fig. 10(a). In contrast, when the suggested algorithm is applied, the symbol points are well arranged as shown in Fig. 10(b). Note the

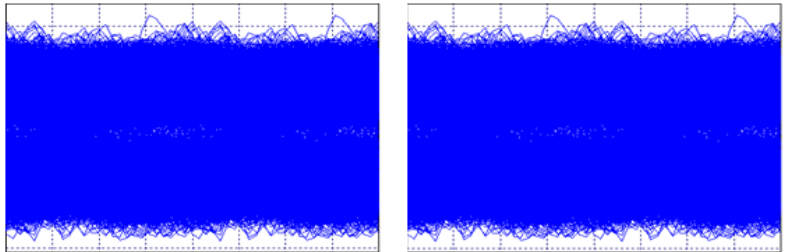

(a)
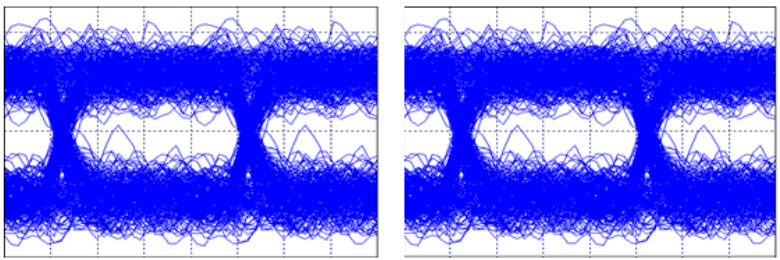

(b)

Fig. 9. Simulated eye diagrams of the I (left) and Q (right) channels for $5 \mathrm{Gbd} / \mathrm{s}$ at B2B; (a) without and (b) with feedback, respectively.

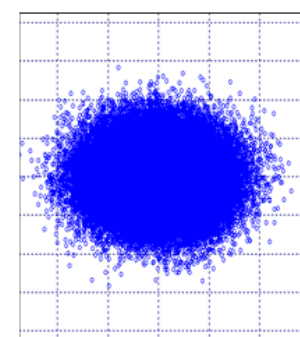

(a)

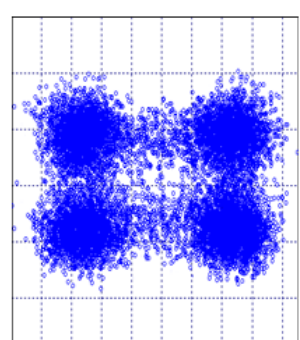

(b)
Fig. 10. Constellation diagrams of the QPSK signals at B2B; (a) without and (b) with feedback, respectively.

similarity between simulation (Fig. 10) and experiments (Fig. 5). In this way we are able to confirm the validity of our simulation results.

Secondly, we transmit the QPSK signal over $10 \mathrm{~km}$ and $25 \mathrm{~km}$, respectively. As shown in Fig. 11, the eye patterns are even more significantly influenced by not only the laser phase noise but also the ASE noise of the EDFA. Hence, the eyes are almost closed as shown in Figs. 11(a) and (c), whereas the eye diagrams are remarkably clear and widely open for both I and Q channels when we apply the suggested method, as shown in Figs. 11(b) and (d). 


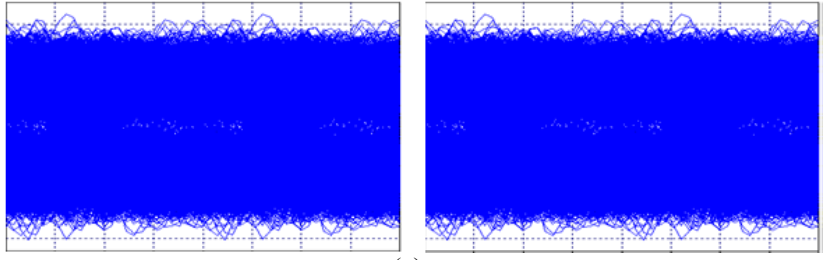

(a)

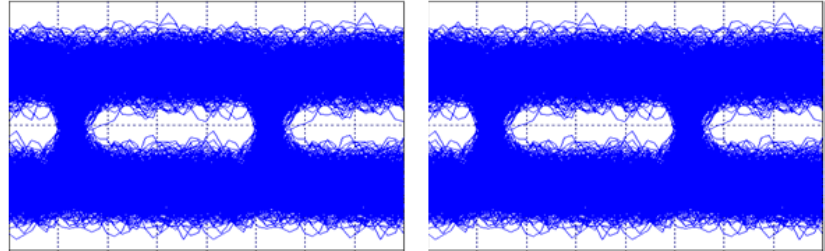

(b)
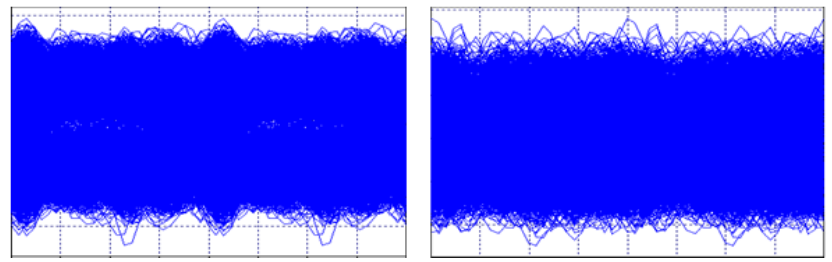

(c)
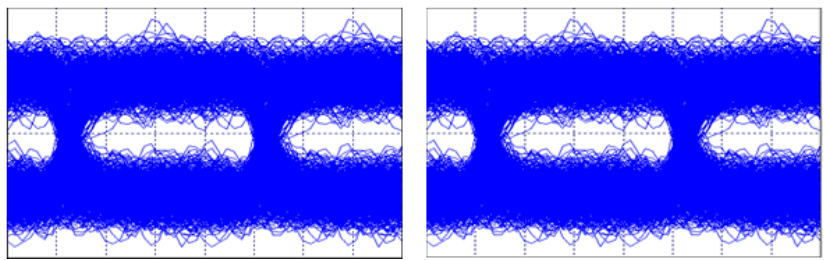

(d)

Fig. 11. Simulated eye diagrams of the I (left) and Q (right) channels for $5-\mathrm{Gbd} / \mathrm{s}$ QPSK signal after $10 \mathrm{~km}$ SMF (a, b) and $25 \mathrm{~km} \mathrm{SMF} \mathrm{(c,} \mathrm{d);} \mathrm{(a,} \mathrm{c)} \mathrm{without} \mathrm{and} \mathrm{(b,} \mathrm{d)}$ with feedback, respectively.

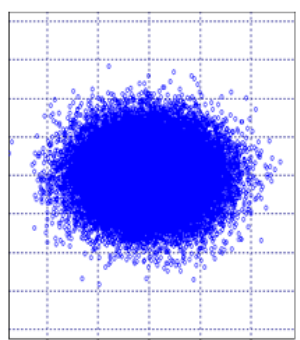

(a)

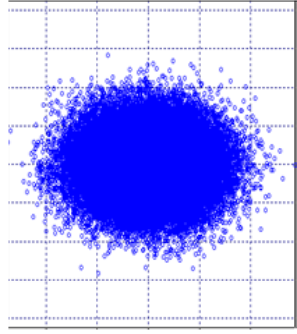

(c)

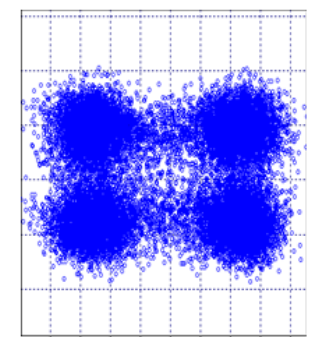

(b)

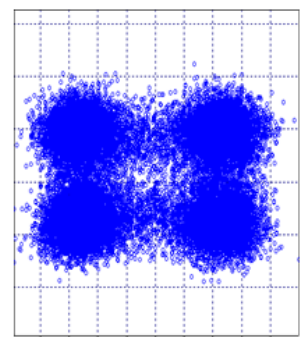

(d)
Fig. 12. Constellation diagrams of QPSK signals after $10 \mathrm{~km}$ $\operatorname{SMF}(\mathrm{a}, \mathrm{b})$ and $25 \mathrm{~km} \mathrm{SMF} \mathrm{(c,} \mathrm{d);} \mathrm{(a,} \mathrm{c)} \mathrm{without} \mathrm{and}$ (b, d) with feedback, respectively.

Figure 12 presents the corresponding constellations of QPSK signals. As expected, the symbol points are blurred and rotated severely, as shown in Figs. 12(a) and (c).
Applying the phase noise compensation method, the symbol points are placed correctly to show rectangular shaped constellation as shown in Figs. 12(b) and (d). Now we can sure that our method is ready for practical application to longer transmission system.

\section{Conclusion}

A novel and simple method, without the aid of DSP, to overcome optical phase noises in a coherent detection system has been suggested. As a proof of the suggested idea, direct coherent demodulation of high-speed optical BPSK/QPSK signal is achieved at real-time by compensating the phase noise through a simple feedback loop. Eye diagrams and corresponding constellations of B2B 5-Gbd/s BPSK and QPSK signals show the remarkable improvement by utilizing the proposed method to compensate the phase noise of the light source. At a $10 \mathrm{~km}$ SSMF transmission link, the phase noises from the laser source and EDFA are also significantly reduced by the suggested method, even though the ISI is enhanced a little bit. Better improvement is expected by reducing the ASE noise from the EDFA and the fiber dispersion. Our simulation results show that the suggested method works well for longer distance transmission of the signals. In principle our method is readily applicable for other high-level modulation formats such as 16-QAM and 64-QAM at arbitrarily high speeds.

\section{Acknowledgments}

This work was supported by the Basic Science Research Program through the National Research Foundation of Korea (NRF) funded by the Ministry of Education, Science and Technology (\#2016R1D1A1B04930224), and by the IITP funded by the Ministry of Science, ICT, and Future Planning (\#2014-3-00501).

\section{References}

[1] KIKUCHI, K. Fundamentals of coherent optical fiber communication. Journal of Lightwave Technology, 2016, vol. 34, no. 1, p. 157-179. DOI: 10.1109/JLT.2015.2463719

[2] LEVEN, A., KANEDA, N., UT-VA KOC, et al. Coherent receivers for practical optical communication systems. In Proceedings of IEEE Conference on Optical Fiber Communication and the National Fiber Optic Engineers Conference. Anaheim (CA, USA), 2007. DOI: 10.1109/OFC.2007.4348697

[3] IP, E., PAK TAO LAU, A., BARROS, D. J. F., et al. Coherent detection in optical fiber systems. Optics Express, 2008, vol. 16, no. 2, p. 753-791. DOI: 10.1364/OE.16.000753

[4] COLAVOlPE, G., FOGGI, T., FORESTIERI, E., et al. Impact of phase noise and compensation techniques in coherent optical systems. Journal of Lightwave Technology, 2011, vol. 29, no. 18, p. 2790-2800. DOI: 10.1109/JLT.2011.2164237

[5] TSUKAMOTO, S., KATOH, K., KIKUCHI, K. Coherent demodulation of optical multilevel phase-shift-keying signals using 
homodyne detection and digital signal processing. IEEE Photonics Technology Letters, 2006, vol. 18, no. 10, p. 1131-1133. DOI: 10.1109/LPT.2006.873921

[6] LY-GAGNON, D.-S., TSUKAMOTO, S., KATOH, K., et al. Coherent detection of optical quadrature-phase-shift-keying signal with carrier phase estimation. Journal of Lightwave Technology, 2006, vol. 24, no. 1, p. 12-21. DOI: 10.1109/JLT.2005.860477

[7] PETROU, C. S., VGENIS, A., ROUDAS, I., et al. Quadrature imbalance compensation for PDM QPSK coherent optical systems. IEEE Photonics Technology Letters, 2009, vol. 21, no. 24, p. 1876-1878. DOI: 10.1109/LPT.2009.2034750

[8] FORESTIERI, E., SECONDINI, M., FRESI, F., et al. Extending the reach of short-reach optical interconnects with DSP-Free direct detection. Journal of Lightwave Technology, 2017, vol. 35, no. 15, p. 3174-3181. DOI: 10.1109/JLT.2016.2647243

[9] HERZOG, F. T. An optical phase locked loop for coherent space communications. Doctoral Thesis. Swiss Federal Institute of Technology, Zurich, 2006.

[10] HA, T. T., SEO, D. S. Direct detection of optical BPSK/QAM without digital signal processing. In Proceedings of $151^{\text {st }}$ the IIER International Conference. Osaka (Japan), 2018, p. 73-75.

[11] HERZOG, F., KUDIELKA, K., ERNI, D., et al. Optical phase locked loop for transparent inter-satellite communications. Optics Express, 2005, vol. 13, no. 10, p. 3816-3821. DOI: 10.1364/OPEX.13.003816

[12] PARK, H. C., LU, M., BLOCH, E., et al. 40Gbit/s coherent optical receiver using a Costas loop. Optics Express, 2012, vol. 20, no. 26, p. B197-B203. DOI: 10.1364/OE.20.00B197

[13] FATADIN, I., SAVORY, S. J., IVES, D. Compensation of quadrature imbalance in an optical QPSK coherent receiver. IEEE Photonics Technology Letters, 2008, vol. 20, no. 20, p. 1733-1735. DOI: 10.1109/LPT.2008.2004630

[14] SAKAMOTO, T., CHIBA, A., KANNO, A., et al. Real-time homodyne reception of $40-\mathrm{Gb} / \mathrm{s}$ BPSK signal by digital optical phase-locked loop. In 36th European Conference and Exhibition on Optical Communication (ECOC). Torino (Italy), 2010. DOI: 10.1109/ECOC.2010.5621234

[15] LEYVA, J. A. L., HIDALGP, C. E. R. Interconnecting university networks using a full-duplex FSO system using coherent detection and polarization-division multiplexing: Design and simulation. In Proceeding of IEEE Optical Interconnects Conference (OI). San Diego (CA, USA), 2015. DOI: 10.1109/OIC.2015.7115702

[16] ZHU, Z., ZHOU, H., XIE, W., et al. 10-Gb/s homodyne receiver based on Costas loop with enhanced dynamic performance. In Proceeding of $16^{\text {th }}$ International Conference on Optical Communication and Networks (ICOCN). Wuzhen (China), 2017. DOI: 10.1109/ICOCN.2017.8121575

\section{About the Authors...}

TrongThuy HA received the Bachelor's degree in Electronics and Communication Engineering from Post and Telecommunication Institute of Technology, HaNoi, VietNam in 2016. He is currently a Master student at the Dept. of Electronics Engineering, Myongji University, Korea. His research interests include photonic processing, digital signal processing for coherent optical communication.

DongSun SEO received the BS and MS degrees in Electronics in 1980 and 1985, respectively from Yonsei University, Korea. In 1989, he obtained the Ph.D. degree in Electrical Engineering from the University of New Mexico, USA. Since 1990 he has been working as a Professor at the Dept. of Electronics, Myongji University, Korea. He published over 80 journal articles and 100 conference papers. His research interests include optical pulse sources, ultrafast optics, high-capacity optical communication, optical processing, optical measurements, and photonics. 